\title{
Modèles d'intégration spatiale et réseau des villes en Europe
} occidentale

Nadine Cattan, Thérèse Saint-Julien

\section{Citer ce document / Cite this document :}

Cattan Nadine, Saint-Julien Thérèse. Modèles d'intégration spatiale et réseau des villes en Europe occidentale. In: Espace géographique, tome $27, n^{\circ} 1,1998$. pp. 1-10;

doi : https://doi.org/10.3406/spgeo.1998.1117

https://www.persee.fr/doc/spgeo_0046-2497_1998_num_27_1_1117

Fichier pdf généré le 02/04/2018 


\title{
Résumé
}

Résumé. - L'article montre comment la mise en réseau des villes européennes est une construction complexe qui se situe à l'articulation de quatre modèles principaux : le modèle centre-périphérie, celui des systèmes urbains nationaux, celui du réseau des "capitales» et, enfin, celui des réseaux spécialisés de villes. Cette dynamique d'intégration du réseau des villes européennes joue sur trois registres, valorisant chacun des logiques spatiales différentes. La première est une logique relationnelle, qui apparaît doublement intégratrice dans la mesure où elle opère à différents échelons géographiques, jouant à la fois sur les effets de la dépendance avec la distance et sur les effets liés aux nouvelles possibilités de connexions. La deuxième est une logique de ressources qui continue d'imposer une mise en valeur différentielle des lieux. La troisième enfin est une logique de maillage qui, à son tour, infléchit les dynamiques.

\begin{abstract}
Abstract. - Models of geographical integration and the Western European urban network. - This paper aims to demonstrate that the networks between the cities of western Europe are complex structures shaped by the combined action of four main models : the core-periphery model, the national urban systems model, the "capital" network model and the specialised urban network model. It goes on to show how the movement towards increased integration of the western European urban network is conditioned by three different spatial logics. The first is a relational logic, comprising the two highly integrative forces of networking based on geographical proximity and networking made possible by new technology. The second is a resources- based logic, which continues to differentiate places. The third is a logic of national administrative divisions, which tends to work against integration.
\end{abstract}




\title{
Modèles d'intégration spatiale et réseau des villes en Europe occidentale
}

\author{
Nadine Cattan, Thérèse Saint-Julien
}

Équipe PARIS, 13 rue du Four, 75006 Paris

\begin{abstract}
RÉSUMÉ. - L'article montre comment la mise en réseau des villes européennes est une construction complexe qui se situe à l'articulation de quatre modèles principaux: le modèle centre-périphérie, celui des systèmes urbains nationaux, celui du réseau des «capitales» et, enfin, celui des réseaux spécialisés de villes. Cette dynamique d'intégration du réseau des villes européennes joue sur trois registres, valorisant chacun des logiques spatiales différentes. La première est une logique relationnelle, qui apparaît doublement intégratrice dans la mesure où elle opère à différents échelons géographiques, jouant à la fois sur les effets de la dépendance avec la distance et sur les effets liés aux nouvelles possibilités de connexions. La deuxième est une logique de ressources qui continue d'imposer une mise en valeur différentielle des lieux. La troisième enfin est une logique de maillage qui, à son tour, infléchit les dynamiques.
\end{abstract}

EUROPE OCCIDENTALE, INTERACTION SPATIALE, MODĖLES, RÉSEAU DE VILLES

Depuis une quinzaine d'années, les études sur les villes européennes se sont multipliées. On dispose depuis peu d'une tentative d'inventaire critique de l'ensemble de ces études (Cattan, 1996). Les projets typologiques ont jusqu'ici largement dominé. En effet, la plupart des travaux ont caractérisé les villes les unes par rapport aux autres indépendamment des propriétés de leur situation géographique. Une cartographie des résultats réintroduit, au mieux, en fin de parcours, les positions géographiques des pôles préalablement caractérisés. Ces travaux, qui ont
ABSTRACT.- Models of geographical integration and the Western European urban network. - This paper aims to demonstrate that the networks between the cities of western Europe are complex structures shaped by the combined action of four main models: the core-periphery model, the national urban systems model, the "capital" network model and the specialised urban network model. It goes on to show how the movement towards increased integration of the western European urban network is conditioned by three different spatial logics. The first is a relational logic, comprising the two highly integrative forces of networking based on geographical proximity and networking made possible by new technology. The second is a resourcesbased logic, which continues to differentiate places. The third is a logic of national administrative divisions, which tends to work against integration.

MODELS, SPATIAL INTERACTION, URBAN NETWORK, WESTERN EUROPE permis de mettre en évidence quelques modèles fonctionnels urbains restent d'intéressants repères. Pourtant, par les cloisonnements qu'elles opèrent, par les limites qu'elles définissent, de telles approches occultent partiellement l'idée de réseau urbain. Rares sont en effet les études qui ont défini le rôle de ces pôles dans la formation d'un espace européen intégré, et identifié les processus qui concourent à cette mise en réseau. Plus rares encore sont les études qui ont fondé théoriquement les dynamiques sur la base desquelles se développe cette mise en réseau. 
On propose une contribution à la modélisation de la mise en réseau des villes européennes, et de la spatialisation associée à cette mise en réseau. Cette contribution s'appuie nécessairement sur une réflexion théorique plus générale, sur la dynamique des réseaux urbains en phase d'intégration forte, qui correspond au passage de systèmes urbains nationaux fortement identifiés à la formation d'un réseau métropolitain européen intégré. Placer l'interaction spatiale entre les pôles urbains au centre de la réflexion est la seule voie qui permet de définir la forme du réseau des villes européennes et les processus de sa transformation. Dans cette perspective, on montrera comment la structuration des villes européennes en un réseau résulte de l'imbrication de plusieurs modèles urbains. On identifiera en outre les logiques qui en sous-tendent la dynamique.

\section{Le réseau des villes européennes: à l'articulation de plusieurs modèles urbains}

La mise en réseau des villes européennes est une construction complexe à l'articulation d'au moins quatre modèles urbains dont les dynamiques se complètent. La perspective de cette proposition n'est pas sans rappeler les travaux de Roger Brunet et de Giuseppe Dematteis. On montrera sur quels points précis cette proposition se démarque de ces contributions essentielles.

\section{Le modèle centre-périphérie}

À la fin des années 1980, avec l'accélération du processus d'intégration européenne, les débats sur la cohésion du territoire européen, sur les nouvelles dynamiques susceptibles de le transformer se sont intensifiés. Cette intensification, largement soutenue par la puissance publique (Union européenne et instances nationales), n'était pas étrangère à la crainte de voir émerger de nouveaux déséquilibres territoriaux et de voir apparaitre des tensions entre ce que serait un «centre» de l'espace européen, premier bénéficiaire du processus, et une périphérie, plus intégrée certes, mais plus dépendante. Les études conduites dans ce contexte ont donc attaché une importance particulière à l'identification et à la signification de ce centre. Sous la diversité des formules, Europe rhénane (Julliard, 1968), dorsale, mégalopole (Gottman, 1961; Brunet, 1989), triangle d'or (Cheshire et al.,
1988), et aussi parfois centre (Cattan, 1992; Brunet, 1989, 1996; Dematteis, 1996), deux notions distinctes ont été parfois confondues.

La première est celle de mégalopole, telle qu'elle a été définie par Gottman, à propos de la côte Est des États-Unis. Elle renvoie à une forme particulière de région urbaine, dans laquelle la dimension morphologique est première. Densités élevées sur de très vastes étendues, relative continuité de l'occupation du sol, concentration exceptionnelle de population, d'activités et de richesse, en un entrelacs complexe de conurbations, de centres mieux individualisés, et d'espaces interstitiels toujours très densément peuplés, sont autant d'attributs attachés à la caractérisation de ce type d'espace. La conjonction de tous ces attributs accroît les potentiels locaux et donc les probabilités d'échanges entre les différents lieux de la région urbaine. Son territoire est fortement intégré, et il a, de ce fait, toutes les chances d'être fonctionnellement très diversifié. La cohésion durable de la mégalopole repose sur le renouvellement permanent de la mise en valeur des ressources humaines, matérielles ou immatérielles que cette région tire de l'étendue, de la densité et de la diversité de sa concentration, et enfin de la qualité des liaisons internes. La mégalopole est avant tout une expression un peu exceptionnelle des forces d'intégration régionale. De tels ensembles régionaux, parfois transnationaux, ont été aussi identifiés au Japon, de Tokyo à Osaka-Kobé, et en Europe.

Chacun s'accorde à reconnaître l'existence d'une mégalopole européenne, vaste dorsale de l'ouest du continent, dont les contours ont été discutés et précisés principalement par Roger Brunet (1989). Pour ce dernier, cette mégalopole, au tracé nord-ouest-sud-est, s'articule sur l'axe rhénan et s'étend du nord de l'Italie au bassin londonien. Nous adoptons ici une acception géographique plus restreinte puisque ni Londres, ni Paris, situés un peu à l'écart, ne font, selon nous, partie intégrante de cette région urbaine. On considère, en effet, que la discontinuité de la trame du peuplement justifie que ces deux métropoles n'y soient pas rattachées. Dans cette région urbaine, le semis des villes grandes et moyennes est exceptionnellement dense, et l'intensité des échanges particulièrement élevée (Cattan $e t$ al., 1994). Si la mégalopole à toutes chances d'intégrer au moins une partie du centre, elle ne se confond pas, pour autant, avec le centre d'un réseau des villes européennes. 
La seconde notion, celle de centre est d'une autre nature. Le centre se définit par rapport à un système territorial, dans lequel il assure les fonctions centrales, liées aux différents domaines d'exercice du pouvoir. Indissociable de sa périphérie, il ne prend sens que dans sa relation dialectique avec elle. Par les polarisations qu'il engendre à grande et à très grande distance, en continuité et en connexité, par les fonctions qu'il concentre, le centre est un puissant moteur, le premier sans doute, de l'intégration territoriale. Sa force d'intégration est directement fonction de sa puissance, de sa capacité d'innovation et d'accumulation. L'interdépendance centre-périphérie pose la question de la place des relations de subordination dans cette interdépendance, de la dissymétrie des échanges, de la capacité de réaction des périphéries aux injonctions centrales, et en particulier de leur aptitude à sécréter des relais qui assurent des relations plus multidirectionnelles et moins dissymétriques.

Le centre est, en Europe, le réseau constitué par un nombre limité de métropoles, pas très éloignées, et en étroite relation les unes avec les autres. Il a une structure polycentrique. Quelles que soient les caractéristiques prises en compte pour identifier ce centre, Londres et Paris en sont toujours, de fait, les piliers. Au-delà, selon les cadres dans lesquels on se place, le centre peut intégrer Amsterdam, Bruxelles, Francfort et Zurich. Verra-t-on, comme d'aucuns le pensent, Berlin rejoindre prochainement cette structure centrale? Londres et Paris exceptés, ces pôles ne sont pas les plus grandes villes de l'ensemble européen, mais les plus grandes dans chacun de leur système urbain national.

Sans pour autant pouvoir être confondus, mégalopole et centre ont une forte intersection. Forte interconnexion entre les éléments, capacité d'innovation élevée sont communs à la mégalopole et au centre. La forte articulation de ces deux derniers doit peu au hasard en Europe. L'extension et la puissance de la mégalopole donnent à certains de ses noyaux toutes les chances de capter en leur faveur une partie des pouvoirs liés aux fonctions centrales, et de se trouver ainsi rattachés au centre. Tirant parti de la proximité du centre, la mégalopole ne peut que profiter des avantages que confère la centralité. À son tour, le potentiel du centre est conforté par la proximité de cette vaste région urbaine.

La force du modèle centre-périphérie dans le processus d'intégration territoriale en Europe n'est plus à démontrer

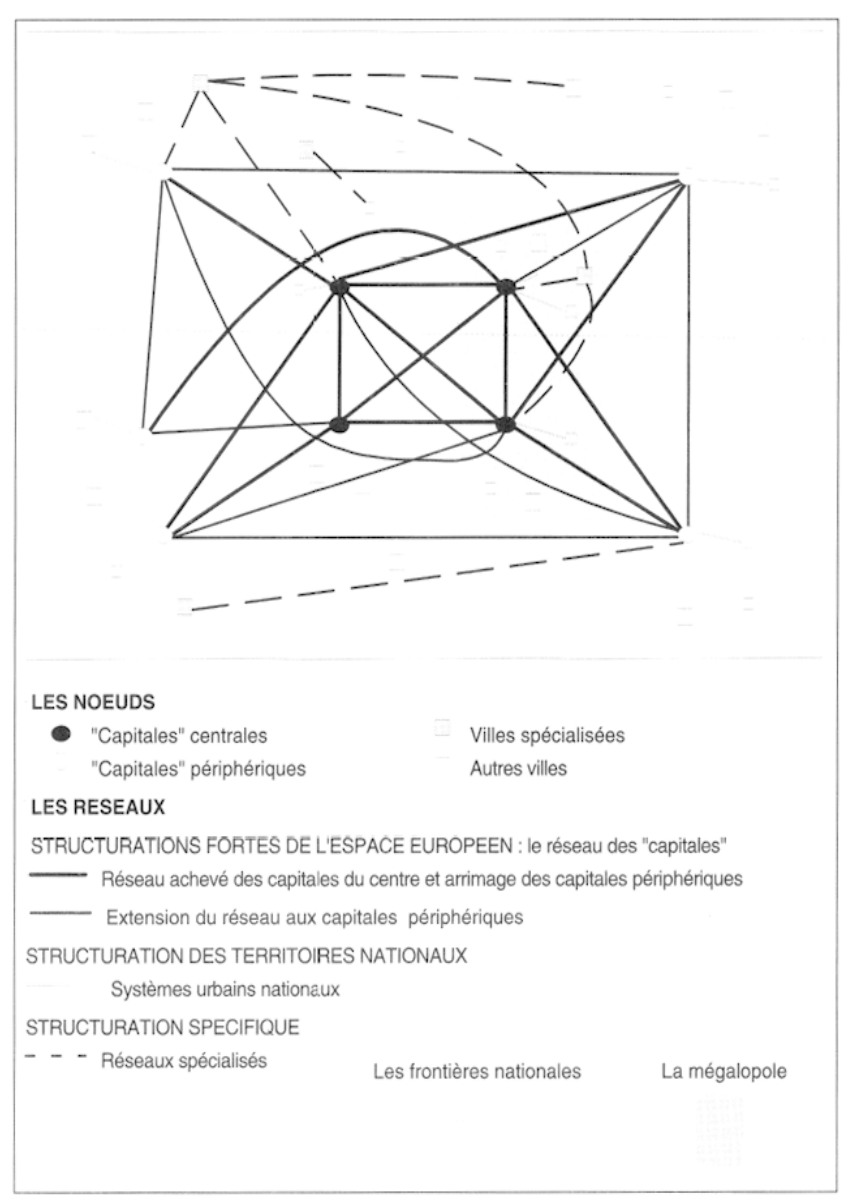

Fig. 1.-Les modèles d'intégration spatiale du réseau des villes européennes.

(Brunet, 1989; Cattan, 1992; Rozenblat, 1992; Brunet, 1996; Dematteis, 1996). Ce modèle n'est cependant qu'un des cadres par lesquels passe l'intégration territoriale $\mathrm{du}$ continent européen.

\section{Les systèmes urbains nationaux}

Jusqu'à une période récente, la représentation des ensembles de villes localisées sur un même territoire national sous la forme de systèmes nationaux de villes était peu contestée. L'observation de l'évolution des ensembles nationaux de villes et celle de la dynamique de leurs interrelations ont conduit à mettre en évidence un fonctionnement en systèmes, auxquels des mécanismes d'auto-organisation conservent une relative stabilité sur la longue durée. Tout s'est jusqu'ici passé comme si, connaissant les positions des villes dans les systèmes urbains nationaux, on pouvait décliner l'essentiel des propriétés qui les caractérisaient. 


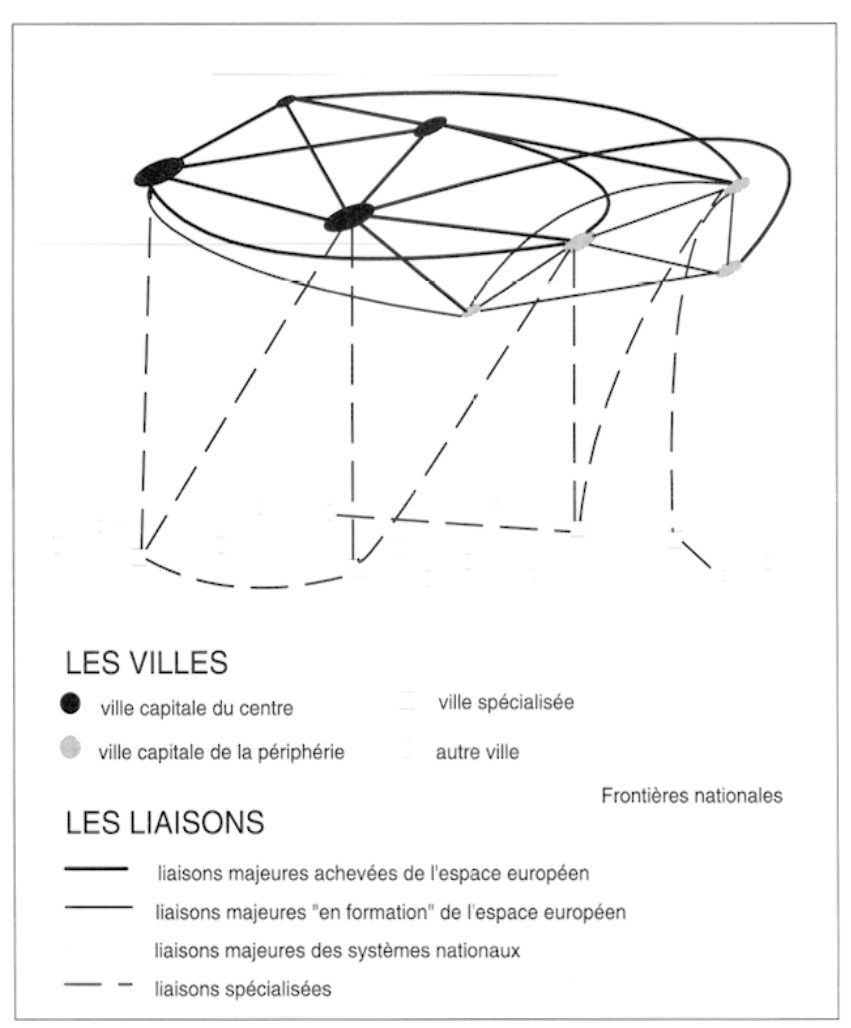

Fig. 2.-L'organisation spatiale du réseau intégré des villes européennes.

Or, depuis quelques années, ce modèle est contesté, en Europe en particulier, au nom de la globalisation, de la diffusion des nouveaux réseaux de communication et plus précisément de l'intégration européenne. Diagnostiquant une obsolescence des cadres nationaux de référence, d'aucuns attirent l'attention sur les réinventions qui s'opèrent à l'échelon local ou, à l'opposé, sur le déplacement des principales forces de structuration territoriale de l'échelon national à l'échelon international. À terme les systèmes nationaux de villes s'affaibliraient inexorablement; ils ne représenteraient plus un échelon pertinent de régulation.

Contrairement à ce que pourraient laisser penser des remarques trop coupées de l'observation, l'échelon des systèmes nationaux de villes est, à court et probablement à moyen terme en Europe, l'échelon qui devrait rester le plus cohérent et le plus structuré. En première approche, l'importance de ce niveau est actuellement de nature à masquer complètement les autres échelons de l'interaction. Quels que soient les échanges interubains que l'on considère, les villes d'un même pays ont tendance, toutes choses égales quant à leur taille et à la distance qui les sépare, à avoir plus de relations entre elles qu'elles n'en ont avec les villes d'un autre pays. Certains travaux ont apporté des précisions éclairantes sur les effets des frontières nationales qui affaiblissent, en particulier, les échanges (Cattan, 1992; Rietveld, 1993). La force des systèmes nationaux est aussi manifeste dans les processus de diffusion spatialc. La propagation de la péri-urbanisation à travers l'ensemble de l'espace européen (Van den Berg et al., 1982; Hall et Hay, 1980), ou encore celle des réseaux de filiation des firmes multinationales (Rozenblat, 1992) sont de bonnes illustrations de la vigueur de cet échelon de structuration.

La bonne résistance des systèmes nationaux de villes n'est pas incompatible avec une augmentation des interdépendances entre les villes à l'échelon européen. L'interdépendance passe par les relais que constituent les capitales, non seulement capitales politiques mais aussi villes qui, bien que n'assurant pas de fonction politique particulière à l'échelon du territoire national, sont des capitales économiques qui exercent sur ce territoire une polarisation incontestée. Cette interdépendance est d'autant plus grande que les systèmes urbains nationaux sont eux-mêmes fortement intégrés. Elle demeure donc tributaire de la cohérence que conservent ces systèmes. Dans un premier temps, le passage par un très petit nombre de relais d'un même territoire pour l'accession au réseau international est de nature à accélérer le processus.

Largement pyramidale, l'articulation des systèmes urbains nationaux à un réseau européen dépend donc des formes des systèmes urbains nationaux. Les systèmes de villes dont la structure est plus hiérarchisée seraient-ils de meilleurs supports pour une mise en réseau généralisée des villes européennes? La réponse à cette question est difficile. Certes, la reprise des concentrations métropolitaines est à peu près partout au rendez-vous en Europe. Les grands opérateurs des transports semblent avoir tranché en faveur d'un modèle radial, et la quasi-totalité des États se préoccupe de la position de leur métropole tête de pont nationale. Autant d'éléments qui militent en faveur d'une réponse positive à cette question. Pourtant, de sérieuses nuances doivent être apportées. D'une part, la forte hiérarchisation de cette mise en réseau est vraisemblablement exacerbée dans cette première phase du processus généralisé d'intégration. Ces effets devraient, par la suite, être en partie corrigés. D'autre part, la modalité pyramidale 
d'intégration n'est pas la seule. D'autres formes de mise en réseau se combinent à elle. Le schéma résultant est beaucoup plus complexe.

\section{Le réseau des villes "capitales»}

Au sens strict, le statut de ville capitale a un fondement politique et administratif. Nous choisissons cependant d'appeler ici réseau des villes «capitales» l'ensemble constitué par toutes les villes capitales politiques de leur État, quand elles se trouvent, au-delà de leurs attributions politiques, en position hiérarchique dominante incontestée dans leur système urbain national (Paris, Londres, Copenhague, par exemple) et aussi les plus grandes métropoles qui, du fait de leur position économique dominante, partagent avec la capitale politique une part de ses attributs métropolitains. Cette situation apparaît plus fréquemment dans les pays où les traditions de décentralisation et de fédéralisme sont vivaces (Francfort, Zurich, Milan, par exemple).

Depuis une quinzaine d'années, l'intensification des échanges entre les villes «capitales» constitutives du centre ou simples éléments de la mégalopole a, certes, progressé relativement plus vite, ce qui n'a pu que conforter la cohésion du centre. Pourtant, le fait majeur demeure le développement des relations entre les capitales plus périphériques, des pays du Sud en particulier. En consolidant leur arrimage au centre, et en les insérant dans le réseau plus large de l'ensemble des villes capitales, cette intensification a progressivement modifié leur position relative dans l'espace européen. Au total, le réseau de l'ensemble des villes capitales constitue le vecteur le plus dynamique de la mise en relation des villes à l'échelon du continent.

En effet, l'intensification des relations entre les villes capitales a fait de ce niveau une dimension forte de l'interdépendance des villes européennes. Cette intensification doit être interprétée à la lumière de l'ensemble des propriétés des situations géographiques de ces capitales. Têtes de pont dans leur propre système urbain national, ces villes sont aussi fortes d'autres caractéristiques de situation qui se valorisent dans l'interaction. Certaines, parties intégrantes de la mégalopole, ont le grand avantage de pouvoir bénéficier du potentiel de ce voisinage. Dans ce contexte régional, ces capitales partagent cependant plus souvent avec d'autres leur rôle de relais: elles apparaissent ainsi plus fréquemment spécialisées. D'autres, capitales de la périphérie, compensent leur éloignement par le fait d'être des relais plus exclusifs pour les systèmes urbains nationaux. D'autres enfin, fortes de leur double fonction de relais national et de centre de l'espace européen, raccourcissent d'autant les circuits de l'intégration pour les villes de leur territoire national.

L'ensemble de ces transformations serait à terme d'une grande portée. Il oriente en effet le réseau des villes européennes vers un modèle plus réticulaire, où la diversité des interconnexions est plus grande. À terme, cette évolution ne manquera pas d'altérer certains traits du modèle centrepériphérie.

\section{Les réseaux spécialisés de villes}

Un quatrième modèle intervient dans le processus d'intégration du réseau des villes européennes. Les villes sont aussi mises en relation par des réseaux spécialisés définis avant tout par une logique de production, que celle-ci soit matérielle où immatérielle. Dans ces réseaux, l'échange peut être à base de complémentarité et de coopération, comme par exemple dans les réseaux financiers, les réseaux de l'industrie aéronautique, les réseaux scientifiques. Les villes les plus spécialisées n'en sont pas les seuls nœuds. Des villes détentrices de portefeuilles diversifiés d'activités peuvent aussi occuper dans ces réseaux des positions dominantes, quand les activités concernées ont localement un poids suffisant. Contrairement aux villes très spécialisées qui, pour leurs relations avec d'autres villes, sont tributaires d'un petit nombre de réseaux, la force des autres villes est de pouvoir participer simultanément au fonctionnement de plusieurs de ces réseaux. L'identification de l'ensemble des réseaux spécialisés, voire de leur fonctionnement, est à ce jour très incomplètement faite. Elle met pourtant en évidence le fait qu'une part des relations interurbaines s'affranchit, pour partie, de contraintes liées, à la fois, à l'organisation hiérarchique des systèmes nationaux et à la distance. Ces réseaux spécialisés démultiplient les articulations entre systèmes urbains nationaux et centre, mais aussi entre systèmes urbains nationaux. L'intensification et la diversification de ces articulations court-circuitent en partie les têtes de pont capitales, accentuant ainsi la connexité du réseau européen des villes. 
Bien qu'ils soient très peu spécialisés, on pourrait reconnaître aux réseaux régionaux qui se constituent sur les zones frontalières un rôle similaire. L'intensification des relations transnationales à moyenne échelle contribue, à la fois, au court-circuitage des têtes de pont nationales et au renforcement de l'intégration des villes européennes par une démultiplication des réseaux de proximité.

\section{Le modèle métropolitain infégré}

L'intégration des villes européennes en un réseau métropolitain intégré résulte de la combinaison de ces quatre modèles. Au total, bien que la mise en réseau des villes européennes soit encore incomplète, on voit se dessiner les traits principaux de cette intégration. Les interdépendances sont particulièrement fortes à deux niveaux, celui des systèmes nationaux de villes d'une part, celui des métropoles qui constituent le centrc de l'espace européen d'autre part. Entre ces deux niveaux les connexions essentielles passent encore majoritairement par les villes «capitales», qui sont, de ce fait, les médiateurs principaux de l'intégration. Si le modèle général se réduisait à cette architecture, la mise en réseau des villes européennes serait fragile et trouverait rapidement sa limite. Le mode de structuration est dans les faits beaucoup plus complexe. D'une part, le réseau des capitales du centre a tendance à s'élargir à l'ensemble des capitales, car les têtes de pont de chacun des systèmes urbains nationaux offrent des chemins d'accès aux autres villes européennes de plus en plus diversifiés. D'autre part, les réseaux spécialisés très nombreux qui, contrairement à certains points de vue hâtifs, concernent un très grand nombre de villes, sont en permanent renouvellement. Ils démultiplient la probabilité de relation entre les villes à l'échelon européen et renforcent la connexité de l'ensemble.

Ce modèle métropolitain intégré retrouve certains des modèles de représentation de l'espace européen de Roger Brunet (1996), et il n'est pas sans rappeler le système urbain métropolitain unifié de Giuseppe Dematteis (1996). Avec le modèle centre-périphérie, on retrouve un élément de structure déjà mis en évidence avec force par chacun de ces deux auteurs. Sans doute faut-il aussi rapprocher le modèle ici proposé du réseau des «capitales » de l'idée d'interconnexion polycentrique par eux évoquée. Toutefois si, avec le modèle polycentrique, Roger Brunet semble se référer implicitement aux métropoles, la notion de réseau interconnecté de Giuseppe Dematteis est multiscalaire et peu dépendante des positions relatives des lieux. Le modèle des réseaux spécialisés n'est pas identifié en tant que tel. Cependant, chaque auteur en intègre l'idée, à sa manière. Roger Brunet évoque la spécialisation à propos des facteurs de mise en réseau que représentent à la fois la «segmentation des filières et la division du travail», et les «relations de compétence». On peut supposer que l'interconnexion multiscalaire de Giuseppe Dematteis est en partie sous-tendue par les effets de la spécialisation interurbaine. Enfin, ni Roger Brunet, ni Giuseppe Dematteis n'identifient les systèmes nationaux de villes comme niveau de régulation interurbaine dans le processus de mise en réseau à l'échelon européen. Plus que tout autre, l'intégration de ce dernier modèle au schéma d'ensemble contribue à individualiser cette proposition.

\section{Les processus de formation du réseau urbain intégré européen}

La dynamique d'un réseau métropolitain intégré dépend d'un certain nombre de processus. Parmi ceux-ci, un petit nombre seulement intègre une forte composante géographique et se trouve dans la dépendance directe des grandes innovations qui redéfinissent en permanence les propriétés des lieux. On en dénombre trois principaux. Le premier est un processus de mise en réseau. Il est lié à une double logique relationnelle. Elle est en premier lieu logique de proximité, contrainte par la distance et l'espacement, et vise à une desserte généralisée du territoire. Elle est en second lieu logique de connexité; elle favorise les concentrations nodales. Le deuxième processus est de mise en valeur différentielle des lieux. Il correspond à une logique de ressources. Le troisième relève d'une dynamique de formation des territoires. Il répond à une logique de maillage.

Ces processus se combinent toujours, mais en des termes qui peuvent varier dans l'espace et dans le temps, l'un pouvant avoir provisoirement avantage sur l'autre. La force de structuration du modèle européen d'un réseau métropolitain intégré relève à la fois de cette combinaison et des temporalités longues de leur développement. 


\section{Mise en réseau : la logique relationnelle}

De tout temps, la mise en relation des lieux a été une condition nécessaire de la formation des réseaux urbains. Cette mise en relation, restée longtemps strictement dépendante de la distance, a fait que, toutes choses égales quant à l'importance des centres urbains, les relations sont d'autant plus intenses que les centres sont proches (principe gravitaire). Au prix de certaines innovations et d'un certain coût, les sociétés se sont employées au cours du temps, à faciliter le franchissement des distances. L'accroissement des vitesses de déplacement, la démultiplication des modes de transport et de communication, la densification des réseaux de relation et l'accès de plus en plus généralisé à ces réseaux ont beaucoup contribué à faciliter ce franchissement. L'ensemble de ces transformations, et surtout leur accélération, ont parfois un peu hâtivement conduit à penser l'évolution d'un système de villes en termes de mutation. Un modèle urbain aux fondements radicalement différents se mettrait en place. D'une suprématie des relations de proximité, on passerait à une domination des relations en connexité, de l'impérieuse nécessité d'une prise en charge par les villes de la continuité de l'espace géographique on passerait à une simple gestion de l'interdépendance des nouds.

Pour séduisante qu'elle soit, une interprétation en termes de rupture conduirait à une vision relativement tronquée des devenirs urbains. Dans les faits, les deux formes d'interdépendance ont de tout temps coexisté. Dans un contexte d'intensification généralisée des échanges à tous les échelons géographiques, la nouveauté vient avant tout du développement beaucoup plus rapide des échanges en connexité, à grande et à très grande distance.

La logique de proximité demeure un des principes actifs de l'unification de tout réseau urbain. Cette logique sous-tend la quasi-totalité des relations banales qui lient les villes entre elles. Par leur masse, ces relations très dépendantes des freins de la distance demeurent un régulateur important de la dynamique des systèmes nationaux de villes, dans la mesure où les exigences de la desserte et de l'encadrement territorial par les villes contribuent à maintenir une trame de lieux centraux. De telles relations ne peuvent donc être considérées comme l'héritage d'un fonctionnement «social» disparu, même si la dynamique en cours simplifie cette trame, ce qui se traduit en particulier par l'obsolescence de niveaux intermédiaires de la hiérarchie des centres.

En Europe, la logique de proximité est, à différentes échelles, un facteur très important de la cohésion du réseau des villes. D'une part, dans un contexte général d'élargissement de la portée géographique des relations, les freins de la distance demeurent suffisamment importants pour que, à l'échelon de l'ensemble du continent, le gradient centrepériphérie continue de jouer un rôle très actif. D'autre part, la forte cohésion des systèmes urbains nationaux, qui s'appuie grandement sur les exigences de la continuité de la desserte, dépend aussi en partie de cette même logique de proximité.

Parallèlement, la logique de connexité, qui intervient de manière croissante dans le processus de mise en relation des villes aux échelons macrogéographiques, affranchit partiellement ce dernier des contraintes de la distance. Une telle logique conduit à deux formes très différentes de mise en relation des lieux. La première, associée à la banalisation des nouvelles technologies de communication de l'information, rend équivalentes les accessibilités des lieux. Elle opère en direction d'une possible reterritorialisation continue de l'espace, dans laquelle la concentration perdrait toute signification et dans laquelle, à terme, les villes ne pourraient que disparaître. Le réseau urbain se dissoudrait dans cette indifférenciation, porteuse de répartitions spatiales, et peut-être en partie sociales, plus égalitaires. On associe souvent à ces nouvelles formes de relations entre les lieux l'image du village planétaire ou encore, celle de «suburbia» généralisée. Cette tendance homogénéisatrice, certes appelée à s'étendre, ne concerne en réalité qu'une partie des échanges immatériels et est, par ailleurs, en permanence contrariée par d'autres formes, tout aussi nouvelles, des relations en connexité.

En effet, loin d'accentuer l'équivalence des lieux, les inégalités d'accès des villes aux réseaux de communication à grandes et très grandes mailles accentuent les concentrations et introduisent de nouvelles hiérarchies des centres urbains. Toutes choses égales quant à leur poids économique, politique, voire symbolique, les villes les mieux placées pour tirer parti des bouleversements des techniques de communication et d'échange sont celles qui occupent, 
simultanément, des positions avantageuses et incontestées dans un très grand nombre de réseaux. De telles villes peuvent développer une part de leurs capacités de polarisation indépendamment de la distance. La force intégratrice de ces villes croît et s'étend du fait de la multiplication des interdépendances qui les lient aux autres villes, à celles qui bénéficient de positions comparables, et à toutes celles qu'elles ont placées dans leur mouvance directe. Si la logique de connexité se déployait de manière isolée, elle ne pourrait que renforcer la concentration sur les nœuds urbains principaux, et ce de manière assez exclusive. L'archipel donne une image forte de ce que serait ce nouveau territoire fragmenté, où joueraient pleinement les effets de ligne et de tunnel, où les processus d'accumulation ne s'opéreraient plus qu'au bénéfice des principaux nœuds de ces nouveaux réseaux.

La logique de connexité, en ce qu'elle accentue les concentrations et les hiérarchies, apparaît en Europe plus déterminante que la logique de proximité dans le processus d'intégration des villes, dans la mesure où ses manifestations les plus spectaculaires sont repérables à l'échelon continental. Elle renouvelle et renforce la cohésion des systèmes urbains nationaux dans les pays de grande dimension, mais elle intervient surtout dans l'articulation de ces systèmes entre eux. L'émergence d'un réseau des villes «capitales», et l'internationalisation des réseaux spécialisés seraient parmi les expressions les plus spectaculaires de sa force. Cette logique tendrait en fait à favoriser le passage d'un réseau hiérarchisé de villes à un système des villes européennes fait d'une hiérarchie de réseaux de villes, qui n'est pas sans rappeler certaines formulations proposées par Roberto Camagni (1993), dont les systèmes nationaux de villes, le réseau des capitales et la structure centrale seraient les pièces maîtresses.

La mise en réseau des villes européennes demeure cependant dans la dépendance directe à la fois de la logique de proximité et de la logique de connexité. Les effets de la continuité du territoire et ceux de la force de la dimension réticulaire des réseaux - dans laquelle le territoire en tant que tel n'est pas pris en compte- devraient pendant longtemps coexister et s'imbriquer. Dans le premier cas, les réseaux à petite et très petite maille assurent l'essentiel des relations; dans le second, au territoire se substitue une structure progressivement réduite à celle des réseaux à grande maille. Dans cette phase de forte intégration territoriale à l'échelon du continent, la logique de proximité contribue au maintien de relations entre les nœuds des réseaux à grande maille et le territoire environnant plus ou moins proche. Même si apparaît une certaine division des relations interurbaines selon les échelons géographiques, les menaces de dislocation sont faibles en Europe, à cause des fortes imbrications de ces deux logiques de relation dans un continent très densément peuplé.

\section{Mise en valeur différentielle des lieux: la logique de ressources}

Les processus inscrits dans des logiques de réseau ne sont pas seuls en cause. Les capacités des lieux à produire et à entreprendre ne sont pas équivalentes. Elles interviennent directement dans l'intégration des villes européennes en un réseau.

Pendant longtemps, la caractérisation des ressources dont disposait une ville relevait le plus souvent d'une dimension matérielle, d'avantages liés au site. L'accent mis sur cette dimension de la ressource n'excluait cependant pas que soient perçues comme telles et exploitées d'autres capacités plus immatérielles tout aussi attachées au site, telles que des capitaux, une culture urbaine, des savoir-faire, des capacités du milieu entrepreneurial, des avantages d'agglomération en tous genres. La définition de la position des villes dans un système urbain, de leur fonction dans cet ensemble, de leur spécialisation, était le plus souvent définie sur la base de la nature du bien produit ou du service vendu. Dans un contexte où l'essentiel des réseaux économiques et techniques s'inscrivait dans un cadre national, et où la division spatiale du travail était assez directement liée à celle des différentes branches d'activité, l'analyse des structures socio-économiques locales et des réseaux d'interdépendance dans lesquels s'inséraient les villes pouvait être réalisée à partir d'une connaissance des biens et des services que celles-ci rassemblaient.

Ce schéma général, certes un peu réducteur, n'a pas disparu. Mais il a dû intégrer des modifications de la notion de ressource urbaine et donc de celle de spécialisation. L'internationalisation des réseaux de production de biens et de services a permis aux pays industriels et, dans ces pays, aux espaces métropolitains, de sélectionner à leur avantage des 
Tabl. 1.-Modèles et processus d'intégration spatiale des villes européennes.

\begin{tabular}{|c|c|c|c|c|c|}
\hline \multirow[t]{2}{*}{ Les processus } & \multirow[t]{2}{*}{ Les logiques } & \multicolumn{4}{|c|}{ Les modèles } \\
\hline & & Centre-périphérie & Systèmes urbains nationaux & Réseaux des villes «capitales» & Réseaux spécialisés \\
\hline \multirow[t]{2}{*}{ Mise en réseau } & Relationnelle de proximité & ++++ & ++++ & 0 & 0 \\
\hline & Relationnelle de connexité & ++++ & ++++ & ++++ & ++++ \\
\hline Mise en valeur différentielle des lieux & Spécialisation & ++ & ++++ & ++++ & ++++ \\
\hline Formation des territoires & Maillage & 0 & ++++ & ++ & 0 \\
\hline
\end{tabular}

++++ Logique majeure ; ++ Logique secondaire ; $\mathbf{0}$ N'intervient pas.

fonctions à la fois dépendantes de la qualité des réseaux de communications et d'échanges internationaux et porteuses des gains de valeur ajoutée les plus élevés. Des périphéries, parfois très lointaines, ont alors accueilli la plupart des segments du processus de production qui pouvaient être satisfaits par un travail peu ou pas qualifié, et mal rémunéré. Dans ces conditions, les ressources immatérielles attachées à un lieu, telles que la qualification des actifs, le niveau général de formation de la population, les capacités du milieu à innover et à entreprendre, la diversité des acteurs ont pris une place de plus en plus grande.

Progressivement, à l'échelon européen, le différentiel de mise en valeur des villes s'est avéré moins bien défini par l'activité que par la nature et le niveau des fonctions assurées dans une activité donnée. Cette évolution, puissant moteur du processus de métropolisation (Pumain, SaintJulien, 1989; Cattan et al., 1994; Veltz, 1996) a eu tendance à donner aux villes qui concentrent les fonctions de conception, d'organisation, de recherche, de recherchedéveloppement et de production d'information une position privilégiée. Les métropoles centrales et plus généralement les «capitales» ont été parmi les premières bénéficiaires de ces nouvelles formes de spécialisation urbaine. Elles ne sont pas les seules. Dans chacun des réseaux nationaux, quelques autres villes d'assez grande taille particulièrement bien dotées, par exemple, en activités de recherche et de recherche-développement, peuvent aussi être concernées, contribuant à la fois à complexifier les réseaux spécialisés existants et à renforcer les échanges avec les métropoles.

La puissance croissante des connexions entre ces centres et la relative immatérialité des ressources donnent à ces nouvelles formes de mise en valeur une certaine instabilité géographique. Cette dernière accentue les tensions entre les villes bénéficiaires, condamnées à coopérer pour maintenir leur position, mais aussi entre centres et périphéries et, en particulier, entre les «capitales» et les autres villes, a fortiori les villes moyennes et les petites. La mise en tension peut enfin être porteuse de ruptures du fait de l'amputation de certains réseaux spécialisés, quand émergent à l'extérieur des réseaux concurrents.

\section{Formation des ferrifoires: la logique du maillage}

On peut être surpris de voir évoquée, à propos de la dynamique d'un réseau métropolitain intégré, la logique de maillage. L'idée de maille ne va-t-elle pas à l'encontre de l'idée d'intégration? Par ses limites, la maille n'introduit-elle pas la notion de barrière, celle de frein des interactions?

Tout, dans la structure du réseau des villes européennes continue de rappeler l'importance des limites des États, leur ancienneté, la dimension des mailles que ces frontières ont fixées, et les effets de barrière qu'elles engendrent. Certaines spécificités de la forme des systèmes nationaux de villes, qui déterminent bien des traits du réseau des villes européennes, ne peuvent être comprises qu'en tenant compte de ce maillage. Les travaux sur le réseau des villes européennes ont bien montré l'importance de cette dépendance. La dimension de la maille étatique joue sur la taille, l'espacement, les fonctions des villes européennes (Julliard et Nonn, 1976; Cattan et al., 1994). La formation des États, associée depuis longtemps à de vastes territoires, a produit des systèmes nationaux de villes plus hiérarchisés comme en France certes, mais aussi en Grande-Bretagne et en Espagne par exemple. En Italie et dans les pays rhénans, où les petits États princiers ont 
survécu beaucoup plus longtemps, la hiérarchie des centres, les partages de fonctions et les espacements des villes s'opèrent sur un mode beaucoup moins inégalitaire. Cette logique de maille joue aussi sur les relations que ces villes entretiennent. Toutes choses égales quant à la taille des villes, la probabilité d'internationalisation de leurs relations est d'autant plus élevée que le territoire national est restreint.

Dans l'individualisation de systèmes nationaux de villes, les frontières des États ont joué un rôle protecteur. On a noté, qu'aujourd'hui encore, les échanges entre grandes villes européennes, qu'il s'agisse des personnes, des marchandises ou de l'information, s'effectuent majoritairement à l'intérieur des limites nationales. Avec la mondialisation des échanges, que l'internationalisation croissante des entreprises ne fait qu'accélérer, l'affaiblissement des barrières que représentent les mailles des territoires nationaux est inéluctable, il ne peut qu'être précipité dans le cadre d'une Europe économiquement et politiquement intégrée. Pourtant, à court et à moyen terme, il faut aussi compter avec toutes les résistances des barrières politiques, sociales, et culturelles, dont ces frontières sont porteuses.

De plus, le rôle de la logique de maillage dans la formation en Europe d'un modèle métropolitain intégré ne saurait être réduit à celui qui revient au maillage national. C'est, au-delà, tout le maillage politico-administratif à l'intérieur des différents États qui est en jeu. Compte tenu du fait bien connu des effets d'entrainement que l'organisation politico-administrative d'un territoire a sur son organisation économique et sur sa structuration culturelle, les effets de ces maillages infranationaux sont à prendre en compte. Ainsi, à l'échelon européen chaque ville du réseau est, entre autres, forte des territoires qu'elle tire avec elle, et ces territoires ne sont pas équivalents (régions, provinces, länder, etc.). En dépit de certains rapprochements des niveaux et des dimensions des mailles infranationales au cours des trente dernières années, les logiques nationales de ces maillages demeurent assez différentes. Elles contribuent au maintien des spécificités des systèmes nationaux de villes.

\section{Références}

BRUNET R. (1989). Les Villes «européennes». Paris: Datar/ Reclus, Documentation française.

BRunet R. (1996). «L'Europe des réseaux ». In Pumain D., Saint-Julien Th., dir. Urban Networks in Europe. Montrouge: John Libbey Eurntext-INED, p. 131-150.

CAmaGNI R. (1993). «Organisation économique et réseaux de villes». In DERYCKE P.H. Espace et dynamiques territoriales. Paris: Economica.

CATTAN N. (1992). La Mise en réseau des grandes villes européennes. Thèse de doctorat, Université Paris I.

CatTan N. (1996). "Comparative studies of sets of European Cities: An overview». In PUMaIN D., SaINT-Jullen Th., dir. Urban Networks in Europe. Montrouge: John Libbey Eurotext-INED.

Cattan N., Pumain D., Rozenblat C., Saint-Julien Th. (1994). Le Système des villes européennes. Paris: Anthropos.

Cheshire P., Hay D., Carbonaro G., Bevan N. (1988). Urban Problems and Regional Policy in the European Community. Luxembourg: Commission of the European Communities.

DemaTTEIS G. (1996). «Towards a unified metropolitan urban system in Europe». In Pumain D., Saint-Julien Th., dir. Urban Networks in Europe. Montrouge: John Libbey Eurotext-INED, p. 19-28.

GotTMan J. (1961). Megalopolis. Cambridge, Mass. : MIT Press.

HallP., HAY D. (1980). Growth Centres in the European Urban System. Londres: Heinemann Educational Books.

Julliard E. (1968). L'Europe rhénane. Paris: A. Colin.

Julliard E., NonN H. (1976). Espaces et régions en Europe occidentale. Paris: CNRS.

Pumain D., Saint-Julien Th. (1989). «La dynamique d'un système de villes». Sistemi Urbani, $n^{\circ} 3$, p. 325-338.

Pumain D., Saint-Julien Th., dir. (1996). Urban Networks in Europe. Montrouge: John Libbey Eurotext/INED, coll. «Congresses and Colloquia».

RIETVELD P. (1993). «International transportation and communication network in Europe: the role of barrier effects». Lyon: Proceedings of the Sixth World Conference on Transportation Research, vol. 2.

ROZENBLAT C. (1992). Le Réseau des entreprises multinationales dans le réseau des villes européennes. Thèse de doctorat, Université Paris I.

SAllez A., dir. (1993). Les Villes lieux d'Europe. Paris: DatarÉditions de l'Aube.

VAN DEN BeRg L., DRewetT R., KLASSEN L.H., RosSI A., VIJVERBERG C.H.T. (1982). A Study of Growth and Decline, Urban Europe, vol. 1. Oxford: Pergamon Press.

Veltz P. (1996). Mondialisation, villes et territoires. L'économie d'archipel. Paris: PUF, coll. «Économie en liberté».

WEGENER M., KUNZMANN R. (1996). «New spatial patterns of european urbanisation». In PUMAIN D., SAINT-JulieN Th., dir. Urban Networks in Europe. Montrouge: John Libbey Eurotext-INED, p. 7-18. 